\title{
Ueber die Identität von Melampyrin und Dulcit;
}

\author{
von Dr. Ludwig Gilmer*).
}

Das von $\mathrm{Hün}$ efeld **) in dem Kraut von Melampyrum nemorosum entdeckte, später von $\mathrm{E}$ i c h l e ${ }^{* * *}$ ) genauer untersuchte und auch in Scrophularia nodosa, sowie in Rhinanthus crista galli nachgewiesene Melampyrin besitzt den Analysen Eich ler's zufolge die Formel $\mathrm{C}_{12} \mathrm{H}_{15} \mathrm{O}_{13}$.

Die Unwahrscheinlichkeit einer solchen Zusammensetzung für einen selbst bei $200^{\circ}$ unveränderlichen Körper veranlafste mich, das Melampyrin einer wiederholten Untersuchung $\mathrm{zu}$ unterwerfen.

Das mir zu Gebote stehende Melampyrin t) bildete schöne weifse Krystallkrusten, und wurde von mir nochmals aus kochendem Wasser umkrystallisirt.

Es veränderte sich nicht bei $100^{\circ}$ und verlor auch in höherer Temperatur nicht an Gewicht. Bei $182^{\circ}$ (uncorrigirt)

*) Auszug aus seiner Inaugural-Dissertation; Tübingen 1862.

**) Journal f., pract. Chemie VII, 233.

***) Jabresber. f. Chemie u. s. w. füx 1856, 665; Chem. Centralblatt 1859 , S. 522 .

†) Es war bezogen von Ernst Merck in Darmstadt. 
schmolz es und erstarrte beim Erkalten wieder krystallinisch. Eich ler giebt den Schmelzpunkt des Melampyrins nur wenig abweichend $\left(186^{\circ}\right)$ davon an.

Beim Erhitzen in einem trockenen Luftstrom im Oelbad fing bei $275^{\circ}$ Wasser zu entweichen an, indem sich dabei die Masse gleichzeitig zu bräunen begann.

Mit Salpetersäure erhitzt lieferte das Melampyrin eine ansehnliche Menge von Schleimsäure. Die von der abgeschiedenen Schleimsäure abfiltrirte Lösung wurde mit kohlensaurem Kalk neutralisirt und filtrirt, worauf die Lösung einen Kupferoxyd in alkalischer Lösung reducirenden Körper enthielt. Der Niederschlag bestand gröfstentheils aus oxalsaurem Kalk, und ich habe vergeblich darin nach Weinsäure oder Traubensäure gesucht.

Bei der Verbrennung des bei $100^{\circ}$ getrockneten Melampyrins mit Kupferoxyd im Sauerstoffstrom erhielt ich folgende Resultate :

I. 0,4800 Grm. Substanz gaben 0,6945 Grm. Kohlensäure und $0,03400 \mathrm{Grm}$. Wasser.

II. 0,2133 Grm. Substanz gaben 0,3105 Grm. Kohlensăure und 0,1504 Grm. Wasser.

In 100 Theilen :

I.

Kohlenstoff

Wasserstoff
39,46

7,87
II.

39,70

7,90 .

Die Resultate lassen zunächst drei Formeln als möglich erscheinen, nämlich : $\mathrm{C}_{10} \mathrm{H}_{12} \mathrm{O}_{10}, \mathrm{C}_{12} \mathrm{H}_{14} \mathrm{O}_{12}$ und $\mathrm{C}_{14} \mathrm{H}_{16} \mathrm{O}_{14}$, da diese sämmtlich nahe zu derselben procentischen $\mathbf{Z u -}$ sammensetzung führen :

\begin{tabular}{|c|c|c|c|c|c|}
\hline $\mathrm{C}_{10}$ & 39,47 & $\mathrm{C}_{12}$ & 39,56 & $\mathrm{C}_{14}$ & 39,62 \\
\hline $\mathrm{H}_{12}$ & 7,89 & $\mathrm{H}_{14}$ & 7,69 & $\mathrm{H}_{\mathbf{1 8}}$ & 7,55 \\
\hline $\mathrm{O}_{10}$ & 52,64 & \multirow[t]{2}{*}{$\mathrm{O}_{12}$} & 52,75 & \multirow[t]{2}{*}{$\mathrm{O}_{14}$} & 52,83 \\
\hline & 100,00 & & 100,00 & & 100,00 . \\
\hline
\end{tabular}


Es bedurfte daher noch einer constant zusammengesetzten Melampyrinverbindung, um die Anzahl der Kohlenstoffäquivalente festzustellen, und erschien hierbei die Barytverbindung am geeignetsten.

Zur Darstellung derselben kochte ich Melampyrin mit Barythydrat und Wasser, wobej ich ungefähr auf 12 Theile Melampyrin 10 Theile krystallisirtes Barythydrat nahm. Die beim Erkalten der filtrirten und luftdicht verschlossenen Lösung sich abscheidenden, wohl ausgebildeten Krystalle wurden mit Weingeist abgewaschen und sogleich im Vacuum über Schwefelsäure getrocknet. Zuletzt wurden sie bis $140^{\circ}$ im Luftbade erwärmt, wodurch alles Krystallwasser sich entfernen liefs.

0,182 Grm. der bei $140^{\circ}$ getrockneten Substanz gaben beim Verbrennen mit chromsaurem Bleioxyd 0,1497 Grm. Kohlensäure und 0,0677 Grm. Wasser.

0,1735 Grm. gaben baim Verbrennen im Platintiegel und nach dem Befeuchten mit Schwefelsïure $0,1320 \mathrm{Grm}$. schwefelsauren Baryt.

Die Analyse führt zu der Formel :

\begin{tabular}{crrc} 
& \multicolumn{2}{c}{ Berechnet } & Gefunden \\
$\mathrm{C}_{12}$ & 72 & 22,7 & 22,4 \\
$\mathrm{H}_{12}$ & 12 & 3,8 & 4,1 \\
$\mathrm{O}_{10}$ & 80 & 25,2 & - \\
$2 \mathrm{BaO}$ & 153 & 48,3 & 49,9 \\
\cline { 2 - 2 } & \multicolumn{1}{c}{317.} & &
\end{tabular}

Die über Schwefelsäure getrockneten Krystalle verloren beim Erwärmen auf $140^{\circ} 20$ pC. Wasser, entsprechend einem Wassergehalt von 9 Aeq. (berechnet $20,3 \mathrm{pC}$. Wasser), womit die Barytbestimmung derselben ebenfalls übereinstimmt.

Es gaben nämlich $0,2915 \mathrm{Grm}$. dor übcr Sehwefelsäure getrockneten Substanz 0,1720 Grm. schwefelsauren Baryt, oder 38,7 pC. Baryt.

0,2370 Grm. gaben ebenso 0,1390 Grm. schwefelsauren Baryt, oder 38,5 pC. Baryt. 
Der Formel $\mathrm{C}_{12} \mathrm{H}_{12} \mathrm{O}_{10}, 2 \mathrm{BaO}+9$ aq. entsprechen 38,4 pC. Baryt.

Die Formel des Melampyrins ist hiernach $\mathrm{C}_{12} \mathrm{H}_{14} \mathrm{O}_{12}$.

Nach diesen Versuchen ist eine Uebereinstimmung in der Zusammensetzung, den Zersetzungsproducten und den Vorbindungen des Melampyrins und des von Laurent Dulcose, von J a cquelain Dulcine, jetzt gewöhnlich Dulcit genannten Körpers, der einmal im Jahr 1848 in grofsen Knollen unbekannter Abkunft von Madagascar nach Paris eingeführt wurde, nicht zu verkennen. Hinsichtlich der physikalischen Eigenschaften ist kein merklicher Unterschied beider Körper zu bemerken. Beide bilden farblose durchsichtige Krystalle, meist zu Krusten vereinigt, von schwach süfsem Geschmack, neutral, mit Hefe nicht gährungsfähig, ohne Rotationsvermögen.

Laure $\mathrm{nt}$ hat die Krystallform des Dulcits bestimmt; er beschreibt sie als monoklinometrische Prismen, deren stumpfe Kanten gerade abgestumpft sind, nebst dem 0ctaëder und der basischen Endfläche $(\infty \mathrm{P} ; \infty \mathrm{P} \infty ;+\mathrm{P} ;-\mathrm{P} ; 0 \mathrm{P})$. Als Kantenwinkel fand Laurent $\infty \mathrm{P}: \infty \mathrm{P}=112^{0}$; $\quad+\mathrm{P}$ : $-\mathrm{P}=115^{\circ} 26^{\prime} ; \infty \mathrm{P}:+\mathrm{P}=134^{\circ} 42^{\prime}$.

Das Melampyrin erhielt ich beim Verdunsten seiner wässerigen Lösung bei gelinder Wärme in ziemlich deutlichen, glänzenden Krystallen. Die beobachteten Flächen waren $\infty \mathrm{P}$; $+\mathrm{P} ;-\mathrm{P} ;+\mathrm{P} \infty ;-\mathrm{P} \infty$ mit den Kantenwinkeln $\infty \mathrm{P}: \infty \mathrm{P}$ $=112^{\circ},+P:-P=115^{\circ} 45^{\prime}$ und $\infty \mathrm{P}:+\mathrm{P}=134^{\circ} 45^{\prime}$. Die Identität der Krystallform beider Körper ist hierdurch erwiesen.

Den Schmelzpunkt des Melampyrins bestimmte ich zu $182^{\circ}$; Laurent giebt den Schmelzpunkt des Dulcits zu $190^{\circ}$, J a c quelain denselben zu $\mathbf{1 8 2}^{\circ}$ an. Beide Stoffe ${ }^{*}$ ) gehen

*) Der von $\mathbf{K}$ ubel (Journal für pract. Chemie LXXXV, 372) aus Evonymus europaeus dargestellte krystallinische Stoff von der Zu- 
aus dem geschmolzenen Zustand beim Erkalten wieder in den krystallinischen über.

Die Zusammensetzung des Dulcits fanden Laurent und Jacquelain der Formel $\mathrm{C}_{12} \mathrm{H}_{14} \mathrm{O}_{12}$ entsprechend. Der Dulcit zerlegt sich beim Erhitzen auf $275^{\circ}$ (Jacquelain), ohne sich dabei bedeutend zu färben.

Mit Salpetersäure erhitzt liefert er Schleimsäure (Laurent); gleichzeitig bildet sich eine Kupferoxyd in alkalischer Lösung reducirende Zuckerart und kleine Mengen von Traubensäure (Carlet)*). Bei dem Melampyrin machte ich dieselben Beobachtungen, doch gelang es mir nicht, Traubensäure nachzuwcisen, wahrscheinlich wegen der geringen Menge des hierzu verwendeten Melampyrins.

Der Dulcit bildet mit den Metalloxyden meist lösliche krystallisirbare Verbindungen. Die leicht krystallisirbare Barytverbindung entspricht nach Laurent der Formel $\mathrm{C}_{12} \mathrm{H}_{12} \mathrm{O}_{10} .2 \mathrm{BaO}$ (bei $170^{\circ}$ getrocknet) und enthält lufttrocken noch 14 Aeq. Krystallwasser. Ich habe die Barytverbindung des Melampyrins nur nach dem Trocknen über Schwefelsäure untersucht, wobei ich 9 Aeq. Wasser fand. Mit Bleizuckerlösung und Ammoniak giebt der Dulcit einen Niederschlag, ebenso das Melampyrin.

Das Melampyrin bildet nach Eichler's Angaben mit Schwefelsüure eine gepaarto Säure, die mit Baryt ein in Wasser lösliches, klebriges oder gummiartiges Salz gieht und durch Silberlösung oder Bleiessig nicht gefällt wird. Ebenso verhält sich der Dulcit nach den Angaben von Jacquelain.

sammensetzung $\mathrm{C}_{12} \mathrm{H}_{14} \mathrm{O}_{12}$, welchen or Evonymit nennt, ist offenbar ebenfalls Dulcit. Den Schmelzpunkt desselben fand Kubel bei $182^{\circ}$, die Krystallform monoklinometriscb.

*) Conpt. rend. LI, 137 und LIII, 343. 
Durch die Vermittelung von E. Merck in Darmstadt kam ich in den Besitz von rohem Dulcit, aus dem ich mir den reinen Körper darstellte. Er war in seinem ganzen Habitus von dem Melampyrin nicht zu unterscheiden.

Um noch einen weiteren Anhaltspunkt zu haben, bestimmte ich die Löslichkeit beider Stoffe in kaltem Wasser.

$24,820 \mathrm{Grm}$. bei $15^{\circ}$ mit Dulcit gesätigter Lösung hinterlicfsen beim Verdampfen $0,771 \mathrm{Grm}$. bei $100^{\circ}$ gotrockneten Rückstand. 100 Theile Wasser von $15^{\circ}$ lösen hiemach nahezu 32 Thl. Dulcit. 29,800 Grm. bei $16^{\circ}$ mit Melampyrin gesättigter Lösung hinter. liefsen 0,984 Grm. trockenen Rückstand.

100 Thl. Wasser von $16^{\circ}$ lösen daher nanezu 34 Thl. Melampyrin.

Beide Körper sind in Weingeist nur in geringer Menge löslich.

Aus dem Angeführten möchte sich zur Genüge ergeben, dafs Melampyrin und Dulcit identisch sind. 